\section{Poster Presentation Abstracts,}

Efficacy of homologous platelet lysate in patients with early knee osteoarthritis

Authors: Ankit Batra ${ }^{1}$, Rajni Ranjan ${ }^{1}$

Affiliation: ${ }^{1}$ Dept. of Orthopaedics, School of Medical Sciences and Research, Sharda University, Greater Noida, Uttar Pradesh, India

Corresponding Author: Ankit Batra

Email: ankitbatra230696@gmail.com

Background: Recently the idea of "Orthobiologics" leads to development of less invasive procedures and accelerated treatments which enhances functional recovery of musculoskeletal disorders. The desired therapeutic effect of autologous platelet rich plasma is facilitated by the ability of IL-1-Ra to limit the destructive inflammatory intra-articular (IA) actions of IL-1 $\beta$. Objectives: To evaluate the efficacy and functional outcome of homologous platelet lysate to reduce pain, improve joint function and enhance quality of life in patients with early knee osteoarthritis and to investigate the advantages and disadvantages of homologous platelet lysate.

Materials and Methods: A total of 40 cases of early knee osteoarthritis were given homologous platelet lysate. Each patient has been given ultrasound guided homologous platelet lysate on day 0 , at the end of 4 weeks and 8 weeks and all the patients were followed for up to one year. Treatment outcome measures were assessed at each visit using VAS and WOMAC scores.

Results: The short-term pain relief observed in both groups are robust in nature and are due to the anti-inflammatory effects of growth factors present in platelets. The long-term pain relief is attributed by improving joint homeostasis and quality of cartilage. The study demonstrated robust improvements in mean outcome in VAS and WOMAC scores \&also demonstrated good treatment effects in $83 \%$ cases at 12 months post-injection. No adverse reactions like swelling, acute pain or infection were noted in the follow up period.

Conclusion: Homologous platelet lysate has the potential to offer chondroprotective and molecular approach in treating pain and functionality in patients with early knee osteoarthritis.

Keywords: Homologous platelet lysate, Osteoarthritis, VAS score, WOMAC score.

Accentuating the sources of MSCs as cellular therapy for osteoarthritis knees - A panoramic review

Author: Arun Gulati ${ }^{1 *}$, Naveen Jeyaraman ${ }^{2}$

Affiliation: ${ }^{1}$ Dept. of Orthopaedics, Kalpana Chawla Government Medical College \& Hospital, Karnal, Haryana, India.

${ }^{2}$ Dept. of Orthopaedics, Kasturba Medical College, MAHE University, Manipal, Karnataka, India.
Corresponding Author: Arun Gulati

Email: gulati_arun123@yahoo.com

The large economic burden on the global health care systems is due to the increasing number of symptomatic osteoarthritis (OA) knee patients whereby accounting for greater morbidity and impaired functional quality of life. The recent developments and impulses in molecular and regenerative medicine have paved the way for inducing the biological active cells such as stem cells, bioactive materials, and growth factors towards the healing and tissue regenerative process. Mesenchymal stem cells (MSCs) act as a minimally invasive procedure that bridges the gap between pharmacological treatment and surgical treatment for OA. MSCs are the ideal cell-based therapy for treating disorders under a minimally invasive environment in conjunction with cartilage regeneration. Due to the worldwide recognized animal model for such cell-based therapies, global researchers have started using the various sources of MSCs towards cartilage regeneration. However, there is a lacuna in literature on the comparative efficacy and safety of various sources of MSCs in osteoarthritis of the knee. Hence, the identification of a potential source for therapeutic use in this clinical scenario remains unclear. In this article, we compared the therapeutic effects of various sources of MSCs in terms of efficacy, safety, differentiation potential, durability, accessibility, allogenic preparation and culture expandability to decide the optimal source of MSCs for OA knee

Keywords: Osteoarthritis, Mesenchymal stem cells, Bone marrow, Stromal vascular fraction, Orthobiologics

\section{Regenerative therapy in delayed union of fractures}

\section{Author: Arunabh Arora ${ }^{1}$, Madhan Jeyaraman ${ }^{1}$}

Affiliation: ${ }^{1}$ Dept. of Orthopaedics, School of Medical Sciences and Research, Sharda University, Greater Noida, Uttar Pradesh, India

Corresponding Author: Arunabh Arora

Email: arunabharora1989@gmail.com.

Introduction: The term delayed union describes an ununited fracture that continues to show progress toward healing or has not been present long enough to satisfy an arbitrary time criterion for nonunion. Recent advances in cellular and molecular biology have led to the identification of specific cytokines that mediate cellular activities which becomes a powerful tool in management of orthopaedic disorders. Here we have tried to observe the functional outcome of autologous platelet rich plasma injection in delayed union of diaphyseal long bone fractures.

Materials and Methods: A total of 20 patients with clinical and radiological criteria of delayed union of fractures were given 3 doses of autologous PRP injection at the interval of 3 weeks per injection. Every patient was followed at regular interval of day 0 , at the end of 3, 6 and 9 weeks $\& 3$ and 6 months for clinical and radiological union of fractures. 
Results: All patients showed hastened union of fracture with excellent functional outcome at the end of $6^{\text {th }}$ month follow up. Discussion: Activated platelets can release more than 300 molecules that are responsible for the coordination of numerous cell-cell and cell-extracellular matrix (ECM) interactions and stimulate cytoskeletal reorganization, chondrocyte and mesenchymal stem cell proliferation and collagen synthesis while diminishing the catabolic effects of inflammatory cytokines.

Conclusion: PRP offers greater advantage in union of fractures by providing micromolecular framework at fracture site. PRP drastically decreases the morbidity of patients by improving the functional quality of life.

Keywords: Delayed union; Platelet rich plasma; Regenerative therapy

Conservative \& physical therapy vs Corticosteroid therapy vs Percutaneous needling with autologous platelet rich plasma therapy for de Quervain's stenosing tenosynovitis

\section{Author: Dushyant Chaudhary ${ }^{1}$, Rakesh Kumar ${ }^{1}$ \\ Affiliation: ${ }^{1}$ Dept. of Orthopaedics, School of Medical Sciences and Research, Sharda University, Greater Noida, Uttar Pradesh, India \\ Corresponding Author: Dushyant Chaudhary \\ Email: dushyantchaudhary41@gmail.com.}

Background: de Quervain's stenosing tenosynovitis (dQSTS) is a tenosynovial disorder which is characterized by impaired gliding of the tendons of the abductor pollicis longus (APL) and extensor pollicis brevis (EPB) muscles over the tendon sheath covering these tendons. The most sensitive clinical test to diagnose dQSTS is Finkelstein manoeuvre. The management of the disease differs based on the severity of the condition.

Objectives: To to prospectively review and compare the efficacy, feasibility and durability of conservative \& physical therapy, corticosteroid therapy and platelet rich plasma therapy in the view of (1) symptomatic pain relief, (2) improved the visual analogue scale (VAS) score and functional Mayo's wrist scores, (3) durability of treatment given and (4) alleviation of need of surgery in patients with de Quervain's tenosynovitis.

Materials and Methods: After screening of cases, 217 cases entered into the study and the cases were randomized into three groups according to our study protocol. Group A cases were treated with conservative \& physical therapy, Group B cases were treated with corticosteroid therapy and Group C cases were treated with autologous platelet rich plasma injection with due pre and post procedural care. The cases were followed up on day 0 , at the end of $1^{\text {st }}$ week, $1^{\text {st }}$ and $6^{\text {th }}$ month for pain and range of movements. The patients are followed up for complications and the data were analysed statistically.

Results: A total of $25(30.06 \%)$ cases in group A, 48 $(69.56 \%)$ cases in group B and $64(95.52 \%)$ cases in group C had recovered from disease at the end of $2^{\text {nd }}$ dose of treatment. At the end of $6^{\text {th }}$ month follow up, a total of 39 $(60.93 \%)$ cases in group A, $21(30.43 \%)$ cases in group B and $3(4.47 \%)$ cases in group $C$ had recurrence. All these patients were followed up for 1 year which showed a statistical difference with $\mathrm{p}$ value of $<0.001$ in VAS score and 0.001 in Mayo's wrist scores among all three groups. No adverse reactions and serious complications are noted in the study participants.

Conclusion: Percutaneous needling with autologous PRP injection is the superior modality for de Quervain's tenosynovitis which minimise the pain and improve the functional quality of life.

Keywords: Platelet rich plasma, De Quervain's tenosynovitis, Corticosteroid, Percutaneous needling

Platelets and Platelet-rich Plasma in Rheumatoid arthritis - A Systematic Review of Literature

Author: Girinivasan Chellamuthu ${ }^{1,}$, Sathish $M$ Muthu $^{1,2,3}$

Affiliation: ${ }^{1}$ Research Associate, Orthopaedic Research Group, Coimbatore, Tamil Nadu, India.

${ }^{2}$ Assistant Professor, Dept. of Orthopaedics, Government Medical College \& Hospital, Dindigul, Tamil Nadu, India.

${ }^{3}$ Research Scholar, School of Engineering \& Technology, Sharda University, Greater Noida, Uttar Pradesh, India.

Corresponding Author: Girinivasan Chellamuthu

Email: giri.c.nivasan@gmail.com

Study Design: Systematic Review

Introduction: The treatment of Rheumatoid Arthritis (RA) has been closely evolving with an understanding of disease pathogenesis with Disease Modifying Anti-Rheumatoid Drugs (DMARDS) and Biologic DMARDS being the main stay. Platelet Rich Plasma (PRP) has been the center of research in many specialties in the past decade. Itsability to stop and reverse inflammation have attracted researchers to try PRP in RA. A systematic review of studies on PRP in RA is lacking.

Objectives: To do a systematic review of literature to summarize the evidences available on the use of PRP in rheumatoid arthritis and also to summarize the role of platelets in the pathology of rheumatoid arthritis.

Materials and Methods: A detailed search of Cochrane, Medline, Embase, and Web of science databases were made to identify the relevant articles till September 2020 following Cochrane and PRISMA guidelines. Number of subjects, Animal model used, cell lines used for the study, method of induction of arthritis, PRP dose, concentration used, frequency of administration and clinical, histologic, and molecular changes from baseline following PRP use were extracted and analysed.

Results: 7 studies were included for the review. Four of these were in-vitro studies. 1 was an exclusive animal study. One study analysed the effects of PRP in RA in both animal models (mice) and Hela cell lines. One study was a report of a series of patients of resistant RA treated with PRP. In the in 
vitro studies while platelets increase the migration and invasion of RA-FLS, they suppressed the inflammation on the whole. Available animal studies and the Human study have shown encouraging results. There has been no evidence of exacerbation of inflammation in these studies.

Conclusion: Available literature is encouraging towards the use of PRP in RA. However, the quantity and quality of literature is limited. Larger trials and molecular studies to understand the exact role of platelets in disease pathogenesis and treatment mechanisms is needed to decide the future course of PRP in RA.

Keywords: Platelet Rich Plasma, Rheumatoid Arthritis, Regenerative Medicine, PRP, Systematic Review, DMARDS

Autologous bone marrow derived mesenchymal stem cell therapy for osteonecrosis of femoral head: A systematic overview of overlapping meta-analyses

\section{Author: Madhan Jeyaraman ${ }^{1}$}

Affiliation: ${ }^{1}$ Dept. of Orthopaedics, School of Medical Sciences and Research, Sharda University, Greater Noida, Uttar Pradesh, India.

Corresponding Author: Madhan Jeyaraman

Email: madhanjeyaraman@gmail.com

Study design: Systematic Review. Objectives: We performed this systematic overview on the overlapping meta-analyses that analysed autologous bone marrow-derived mesenchymal stem cell (BM-MSC) therapy along with core decompression (CD) for the management of osteonecrosis of the femoral head $(\mathrm{ONFH})$ and identify which study provides the current best evidence on the topic and generate recommendations for the same. Materials and methods: We conducted independent and duplicate electronic database searches in PubMed, Web of Science, Embase, Cochrane Database of Systematic Reviews, and the Database of Abstracts of Reviews of Effects till September 2020 for meta-analyses that analyzed the efficacy of BM-MSC therapy along with CD for ONFH. Methodological quality assessment was made using Oxford Levels of Evidence, AMSTAR scoring, and AMSTAR 2 grades. We then utilized the Jadad decision algorithm to identify the study with the highest quality to represent the current best evidence to generate the recommendation. Results: 6 meta-analyses fulfilling the eligibility criteria were included. The AMSTAR scores of the included studies varied from 4 to 9 (mean:7) and all the included studies had critically low reliability in their summary of results due to their methodological flaws according to AMSTAR 2 grades. The current best evidence showed that utilization of BM-MSC therapy along with $\mathrm{CD}$ for $\mathrm{ONFH}$ resulted in significant improvement in Harris hip scores at 12 and 24 months along with a significant reduction in the necrotic area of the femoral head and the rate of conversion to total hip arthroplasty (THA) without a significant rise in adverse events due to the procedure. Conclusion: Based on this systematic overview, we give a Level II recommendation that BM-MSC therapy is more efficacious along with $\mathrm{CD}$ in the management of ONFH compared to $\mathrm{CD}$ alone. BM-MSC therapy provides better pain relief with significant functional improvement and delaying the collapse of the femoral head thereby preventing further treatment such as THA.

Keywords: Osteonecrosis, Core Decompression, Mesenchymal Stem Cell

A biological therapeutic option for retrocalcaneal bursitis and its associations.

Author: Naveen Jeyaraman ${ }^{1}$, Prajwal GS ${ }^{2}$

Affiliation: ${ }^{1}$ Dept. of Orthopaedics, Kasturba Medical College, MAHE University, Manipal, Karnataka, India.

${ }^{2}$ Dept. of Orthopaedics, JJM Medical College, Davangere, Karnataka, India.

Corresponding Author: Naveen Jeyaraman

Email: naveenjeyaraman@yahoo.com

Background: Retrocalcaneal bursitis is the inflammation of the bursa between the anterior aspect of the Achilles and posterior aspect of the calcaneum which is associated with achilles tendinitis, plantar fasciitis, calcaneal spurs, fibromyalgia and rheumatoid arthritis.

Objectives: This study is conducted to review a series of patients prospectively with symptomatic retrocalcaneal bursitis and its associations to determine if PRP injections (1) provide symptomatic relief, (2) improved VAS and AOFAS scores and (3) alleviation of need of surgery.

Materials and Methods: After screening of cases, 128 cases entered into the study and have been treated with an autologous platelet rich plasma injection with due pre and post procedural care. The cases are followed up on day 0 , at the end of $1^{\text {st }}$ week, $1^{\text {st }}$ and $6^{\text {th }}$ month for pain and range of movements. The patients are followed up for complications and the data were analysed statistically.

Results: Out of 128 cases, 76 patients (59.37\%) improved with $1^{\text {st }}$ dose and further 38 patients $(29.68 \%)$ with $2^{\text {nd }}$ dose of autologous PRP injection with an interval of 3 weeks from the first dose. A total of $89.05 \%$ of patients, who got treated with autologous PRP injection, had a good clinical and functional outcome even at the end of 1 year of injections and presented with statistically significant results with $\mathrm{p}$ value < 0.001 . No adverse reactions and serious complications are noted in the study participants.

Conclusion: The autologous PRP injection is considered superior in treatment of retrocalcaneal bursitis which minimise the pain and improve the functional quality of life.

Keywords: Platelet rich plasma, Plantar fasciitis, Retrocalcaneal bursitis, Achilles tendinitis.

Mesenchymal Stem Cell-Derived Exosomes: A Potential Therapeutic Avenue in Knee Osteoarthritis

Author: Prajwal GS ${ }^{1}$, Ramesh $\mathbf{R}^{1}$ 
Affiliation: , ${ }^{1}$ Dept. of Orthopaedics, JJM Medical College, Davangere, Karnataka, India.

Corresponding Author: Prajwal GS

Email: prajwalgs1894@gmail.com

Knee osteoarthritis is the leading cause of functional disability in adults. The goals of knee osteoarthritis management are directed toward symptomatic pain relief along with the attainment of the functional quality of life. The treatment strategy ranges from conservative to surgical management with reparative and restorative techniques. The emergence of cell-based therapies has paved the way for the usage of mesenchymal stem cells (MSCs) in cartilage disorders. Currently, global researchers are keen on their research on nanomedicine and targeted drug delivery. MSCderived exosomes act as a directed therapy to halt the disease progression and to provide a pain-free range of movements with increased quality of cartilage on regeneration. International Society for Extracellular Vesicles and the European Network on Microvesicles and Exosomes in Health and Disease have formed guidelines to foster the use of the growing therapeutic potential of exosomal therapy in osteoarthritis. Although regenerative therapies with MSC are being seen to hold a future in the management of osteoarthritis, extracellular vesicle-based technology holds the key to unlock the potential toward knee preservation and regeneration. The intricate composition and uncertain functioning of exosomes are inquisitive facets warranting further exploration.

Keywords: Mesenchymal stem cells, Exosomes, Microvesicles, Cartilage

\section{Functional outcome periarthritis shoulder treated with autologous PRP injections}

\section{Author: Rashmi Jain ${ }^{1}$, Madhan Jeyaraman²}

Affiliation: ${ }^{1}$ School of Medical Sciences and Research, Sharda University, Greater Noida, Uttar Pradesh, India

${ }^{2}$ Dept. of Orthopaedics, School of Medical Sciences and Research, Sharda University, Greater Noida, Uttar Pradesh, India

Corresponding Author: Madhan Jeyaraman

Email: madhanjeyaraman@gmail.com

Background: Periarthritis shoulder is also called frozen shoulder which describes a chronic, indolent pathological process in which the body forms excessive adhesions across the glenohumeral joint which in turn leads to pain, stiffness, and loss of range of movements which compromises the quality of life. The histological biopsy of the contracted capsule revealed the deposition of fibroblasts admixed with type 1 and 3 collagen where there will be a transformation of fibroblasts into myofibroblasts with altered levels of matrix metalloproteinases. The management of periarthritis shoulder ranges from non-operative management to surgical release of fibrosis of shoulder joint. This study is to evaluate the functional outcome of autologous PRP injections in periarthritis shoulder.

Materials and Methods: After excluding the patients who failed to satisfy the study protocol, the remaining 60 patients of periarthritis shoulder with a single dose of autologous PRP injection and were followed up pre-procedurally and postprocedurally at the end of $1^{\text {st }}, 6^{\text {th }}$ and $12^{\text {th }}$ month for pain relief and range of movements. The improvements in pain and range of movements are charted in terms of VAS and DASH scoring system.

Results: A total of $72 \%$ of patients reported better pain control at 6 months with $90 \%$ of patients reporting improvement at 12 months. A statistically significant improvement $(p<0.045)$ is observed with VAS $(p<0.01) \&$ DASH score $(\mathrm{p}<0.03)$ in periarthritis shoulder patients over 12 months. At the end of 6 months, $72 \%$ of patients had better pain control with good range of shoulder movements. 7 patients has lost follow up during the study period. No adverse reactions like swelling, acute pain or infection were noted.

Conclusion: Platelet rich plasma injections have proved in reducing pain, improve joint motion \& function and enhance quality of life in patients. For periarthritis shoulder, autologous PRP therapy remain functionally superior as autologous PRP is a constructive procedure by rejuvenating the degenerative tissues.

Keywords: Platelet rich plasma, Frozen shoulder, Periarthritis.

\section{Role of intradiscal injection of Platelet Rich Plasma in the management of Lumbar Disc Disease Systematic Review \& Meta-analysis}

\section{Author: Sathish Muthu ${ }^{1,2,3}$ Girinivasan Chellamuthu ${ }^{2}$}

Affiliation: ${ }^{1}$ Assistant Professor, Dept. of Orthopaedics, Government Medical College \& Hospital, Dindigul, Tamil Nadu, India.

${ }^{2}$ Research Associate, Orthopaedic Research Group, Coimbatore, Tamil Nadu, India.

${ }^{3}$ Research Scholar, School of Engineering \& Technology, Sharda University, Greater Noida, Uttar Pradesh, India.

Corresponding Author: Sathish Muthu

Email: drsathishmuthu@gmail.com

Study Design: Systematic Review \& Meta-analysis.

Objectives: We performed this meta-analysis to evaluate whether intradiscal Platelet Rich Plasma(PRP) injection has any beneficial role in the management of lumbar disc disease. Materials and Methods: We conducted independent and duplicate electronic database searches including PubMed, Embase, and Cochrane Library till September 2020 for studies investigating the role of intradiscal PRP in the management of lumbar disc disease. The analysis was performed in the $\mathrm{R}$ platform using OpenMeta[Analyst] software.

Results: 13 studies including 2 RCTs, 5 prospective, and 6 retrospective studies involving 319 patients were included in 
the meta-analysis. A single-arm meta-analysis of the included studies showed a beneficial effect of the intervention in terms of pain relief outcomes like VAS score $(\mathrm{p}<0.001)$, pain component of SF-36 ( $\mathrm{p}=0.003)$ while such improvement was not seen in functional outcome measures like ODI score $(\mathrm{p}=0.071)$, the physical component of SF-36 $(\mathrm{p}=0.130)$ with significant heterogeneity noted among the included studies. No structural improvement in magnetic resonance imaging was observed $(\mathrm{p}=0.106)$. No additional procedure-related adverse events were noted in the included studies $(\mathrm{p}=0.662)$. Conclusion: There is a paucity of high-quality studies to give conclusive evidence on the benefits of intradiscal PRP for lumbar disc disease. Although intradiscal PRP injection has shown some beneficial effect in controlling pain for lumbar disc disease, we could not find structural or functional improvement from the included studies. Hence, we recommend large double-blind double-arm randomized controlled studies to analyze the benefits of the intervention being analyzed.

Keywords: Platelet Rich Plasma, Meta-analysis, Lumbar Disc Disease, Regenerative Medicine, Back Pain, PRP

\section{Translational research in chronic tendinopathies-From bench to bedside applications}

\section{Author: Vijay J. Patel ${ }^{1}$, Sathish Muthu ${ }^{2,3,4}$}

Affiliation: ${ }^{1}$ Fellow in Orthopaedic Rheumatology, Dr Ram Manohar Lohiya National Law University, Lucknow, Uttar Pradesh, India.

${ }^{2}$ Assistant Professor, Dept. of Orthopaedics, Government Medical College \& Hospital, Dindigul, Tamil Nadu, India.

${ }^{3}$ Research Scholar, School of Engineering \& Technology, Sharda University, Greater Noida, Uttar Pradesh, India.

${ }^{4}$ Research Associate, Orthopaedic Research Group, Coimbatore, Tamil Nadu, India.

Corresponding Author: Vijay J. Patel

Email: drvjy2012@gmail.com

Chronic tendinopathies involve majority of patients in clinical practice of orthopaedic surgeons and sports physicians. Translational medicine confers an emerging medical advance efficiently towards the clinician directly from scientists which may be used as a targeted therapy. The main objective of translational research from "bench to bedside" is to test novel inventions in humans. Our purpose in this article is to understand the translational medicine approach for chronic tendinopathies in clinical aspects. Translational research in chronic tendinopathies is required certainly due to plenty of reasons. Newer advances and targeted approach to these tendon disorders may curtail the further degenerative process. It aids in earlier diagnosis and prevention of morbidity, early recovery to occupational activity, lack of economical as well as recreational failure. Pre-disease level activity is ultimate goal of any therapy. Tendon pathophysiology is constantly evolving researched topic in both biochemical as well as molecular aspect. The basic fundamental understanding of the process of tendon healing and its regeneration is necessary for formulating a newer guideline. The cornerstone of treatment of tendinopathies is still non-operative management. Physical therapy, better pain control, NSAIDS are still primary choice for these conditions. Various biological therapy whenever used one should combine them with other appropriate options to obtain an optimal outcome.

Keywords: Tendinopathies, Translational Research, Biological therapy, Regenerative Medicine, Inflammation, Sports Medicine 